\title{
Noch einige Worte über die Elimination des Quecksilbers nach der Injektion schwerlöslicher Quecksilberpräparate.
}

Von

Prof. Edvard Welander, Stockholm.

(Hiezu Taf. V-IX.)

In einem Aufsatz in diesem Archiv 1908 „Über Quecksilberbestimmungen im Urin" hat Fräulein Ratn er über einige Untersuchungen berichtet, die sie in Bürgis Laboratorium über die Elimination von $\mathrm{Hg}$ nach der Injektion sogenannter unlöslicher Quecksilberpräparate ausgeführt hat. Sie ist zu genau demselben Resultate, wie Bürgi, gekommen, nämlich daß der Eliminationstypus für Quecksilber für Injektionen von Salizylquecksilber, auch für (essigsaures) Thymolquecksilber und Kalomel (Ol. mercurioli hat sie nicht untersucht) gilt, d. h. daß am ersten Tage nach einer Injektion dieser Mittel eine sehr bedeutende Elimination von $\mathrm{Hg}$ stattfindet, welche Quantität sich während der darauf folgenden $(2-3)$ Tage bedeutend vermindert.

Eine bedeutende Elimination von $\mathrm{Hg}$ während des ersten Tages nach der Injektion dieser Mittel ist gleichbedeutend mit einer schnellen und kräftigen Absorption von $\mathrm{Hg}$ nach einer solchen Injektion, was natürlich in praktischer Beziehung von großer Bedeutung ist.

Tausende Quecksilberuntersuchungen, die ich seit dem Jahre 1885 mit verschiedenen Hg-Präparaten und bei verschie- 
denen Anwendungsmethoden von $\mathrm{Hg}$ vorgenommen habe und wobei ich die Elimination des Quecksilbers mit dem Resultate der Behandlung verglichen habe, haben mir gezeigt, daß als allgemeine Regel aufgestellt werden kann, daß, je schneller das $\mathrm{Hg}$ bei einer $\mathrm{Hg}$-Behandlung absorbiert wird, um so schneller auch die Symptome verschwinden, ebenso, daß, je schneller und kräftiger die Absorption von $\mathrm{Hg}$ bei dieser Behandlung geschieht, um so kürzer die Remanenz des $\mathrm{Hg}$ ist, wenigstens in einer solchen Quantität, daß man sich eine therapeutische Wirkung versprechen kann, weshalb man nach einer solchen Behandlung eine anhaltende Wirkung des $\mathrm{Hg}$ in präventiver Beziehung nicht erwarten kann. Andererseits hat die Erfahrung mich gelehrt, daß , je langsamer das $\mathrm{Hg}$ absorbiert wird, um so langsamer die Symptome der Syphilis verschwinden, während bei einer solchen Hg-Behandlung das Quecksilber lange im Organismus remaniert, weshalb man das Recht hat, nach dieser eine lange Zeit eine Wirkung des $\mathrm{Hg}$ in präventiver Beziehung $\mathrm{zu}$ erwarten und anzunehmen, daß hierdurch dem Auftreten wirklicher Rezidive lange vorgebeugt werden kann. Besonders gilt dies für die angeführte Injektionsbehandlung mit löslichen und mit den obengenannten schwerlöslichen Hg-Präparaten.

Wären nun $\mathrm{B} \ddot{u} \mathrm{rg}$ is Ansichten richtig, so hätte man das Recht, nach der Injektion von Thymolquecksilber, Kalomel (er. 0l. mercurioli) eine gleich schnelle und kräftige Einwirkung auf das Verschwinden der syphilitischen Symptome zu erwarten, wie nach der von Salizylquecksilber; man hätte das Recht zu erwarten, daß nach der Injektion sämtlicher dieser Mittel die Wirkung in präventiver Beziehung gleich lang und ron sehr kurzer Dauer sei.

Meine Erfahrungen hierüber stimmen nicht mit den Untersuchungen Bürgis und Frl. Ratners überein. Meiner Erfahrung nach verschwinden die syphilitischen Symptome nach der Injektion von Salyzylquecksilber in der Regel rasch, nach der Injektion von Thymolquecksilber und Kalomel indessen ziemlich langsam und nach der Injektion von Ol. mercurioli noch langsamer. Dagegen treten nach abgeschlossener Injektionskur mit Sal. Hg sehr bald Rezidive ein (im vergangenen Jahre hatte ich mehrmals Gelegenheit, dies zu beobachten), 
während die Behandlung mit Thymol-Hg und Kalomel, sowie noch mehr mit 0l. mercurioli das Auftreten von Rezidiven in der Regel für längere Zeit verhindert.

In vollkommener Übereinstimmung hiermit gehen auch meine Untersuchungen über die Elimination des Quecksilbers und die Absorption nach der Injektion mit diesen Mitteln. Nach der Injektion von Sal.-Hg erfolgt am ersten Tage nach der Injektion eine sehr bedeutende Elimination von $\mathrm{Hg}$; in den folgenden Tagen nimmt die Elimination stark ab. Bei der Injektion von Thymol-Hg und Kalomel sowie von Ol. mercurioli tritt eine allmählich steigende Elimination von $\mathrm{Hg}$ - am geringsten während des ersten Tages - ein; nach der Injektion von Ol. mercur. steigt die Absorption (resp. die Elimination) langsamer als nach der Injektion der beiden anderen $\mathrm{Hg}-$ Präparate, d. h. mit anderen Worten, der Eliminationstypus für Sal.-Hg ist ein von dem Eliminationstypus für Thymol-Hg, Kalomel und 0l. mercurioli ganz verschiedener. Ebenso haben meine Untersuchungen mir gezeigt, daß die Remanenz des $\mathrm{Hg}$ in nennenswerter Menge nach der Injektion von Sal.-Hg eine kurze ist, während die Remanenz nach der Injektion der anderen obengenannten Hg-Präparate, besonders des Ol, mercurioli eine lange ist.

Nun hat Frl. Ratner gegen den Wert meiner Untersuchungen opponiert und betreffs dieser gesagt, daß die von mir angewendete Almén-Schillberg sche Methode „nur dem Scheine nach quantitativ ist", obschon sie nicht einmal den Versuch gemacht hat, die Methode zu prüfen. Betreffend die verschiedenen Resultate ihrer und meiner Untersuchungen nach dem Farupschen Verfahren, welche letzteren von einem sehr bedeutenden Chemiker in Stockholm, Apotheker B10mqvist, ausgeführt sind, erklärt Frl. Ratne r ganz kurz, daß das Resultat seiner Untersuchungen nauf Fehler der Analyse zurückzuführen ist", welches Urteil sie erst eingehender beweisen sollte.

Da nun diese Frage der Elimination des Quecksilbers nach der Injektion dieser verschiedenen schwerlöslichen ${ }^{\circ} \mathrm{Hg}$ Präparate eine praktische Bedeutung hat, will ich nicht unterlassen, weitere von mir nach verschiedenen Richtungen hin gemachte Untersuchungen vorzulegen, die darauf hindeuten, 
daß ich Grund zu der Behauptung gehabt habe und weiter habe, daß die Resultate meiner Untersuchungen richtig sind, trotzdem sie nicht mit denen Bürgis und Frl. Ratners übereinstimmen.

Über die Möglichkeit, sich mittels der Almén-Schillbergschen Methode eine Vorstellung von der Quantität eliminierten Quecksilbers, ob sich eine kleine oder eine bedeutende Menge $\mathrm{Hg}$ in einem Urin befindet, zu machen, will ich hier nicht ins Detail eingehen, sondern nur auf meinen Aufsatz über die Absorption und Elimination des Quecksilbers, p. 14 u. 15 im Nord. Med. Archiv 1886, verweisen. Ich will hier nur bemerken, daß, wenn man bei der Untersuchung von Urin auf $\mathrm{Hg}$ in dem einen Falle unter dem Mikroskop nur ein paar oder einige sehr kleine Hg-Kugeln entdecken kann und in dem anderen eine höchst bedeutende Menge größerer und kleinerer solcher Kugeln findet. daß, sage ich, die gesunde Vernunft einem sagen muß daß der letztere Urin eine bedeutend größere Menge $\mathrm{Hg}$ enthalten muß, als der erstere. ") Auf Grund der Größe und Menge der $\mathrm{Hg}$-Kugeln teilte ich auch schon 1885 die $\mathrm{Hg}$-Menge in gering, groß, bedeutend usw. ein, um eine approximative Vorstellung von der Größe der eliminierten HgQuantität, z. B. im Urin von auf verschiedene Weise behandelten Personen, geben zu können. Ich betonte, daß gegen diese quantitative Schätzung der Quecksilberquantität natürlich Einwürfe gemacht werden können und daß diese nur einen relativen Wert hat, was jedoch nicht hindert, daß sie im großen ganzen praktisch gesehen genügt, uns eine ganz hinreichende Vorstellung davon zu geben, ob eine größere oder kleinere Quantität $\mathrm{Hg}$ eliminiert resp. absorbiert worden ist.

Natürlich können genauere quantitative Untersuchungen auf $\mathrm{Hg}$ im Urin uns eine sichere Auffassung in diesen Fragen geben; deshalb habe ich auch bei den in diesem Archiv 1907

1) Ich bemerke, daß es auch einem mit der Methode ungewohnten Untersucher keine Schwierigkeit macht, qualitativ nachzuweisen, daß sich z. B. im Urin $\mathrm{Hg}$ findet, sofern die Quantität nicht allzu klein ist, daß aber eine große Übung und eine große Genanigkeit daza gehört, diese Untersuchungen so auszuführen, daß man berechtigt ist, über die approximative Quantität $\mathrm{Hg}$ im Urin Schlüsse zu ziehen. 
Noch einige Worte üb. d. Elimination des Quecksilbers etc. 167

in einem Aufsatz: „Zur Frage der Behandlung der Syphilis mit Injektion von Salizylsäure-Quecksilber und mit Merkurialöl“ veröffentlichten Untersuchungen das Farupsche Verfahren angewendet.

Eine auch nur teilweise Verurteilung der Farupschen Methode, Hg im Urin nachzuweisen, habe ich nicht ausgesprochen, wie es Frl. Ratner behauptet, ich habe aber gesagt, daß man auch mit der Farupschen Methode betreffs der Absorption, Elimination und Remanenz des Quecksilbers nur zu approximativen Werten, obschon in exakten Zahlen ausgedrückt, kommen kann; denn behufs einer exakten Beurteilung desselben müssen wir nicht nur Urin, Schweiß, Speichel usw., sondern vor allem auch die Faeces und zwar eine längere Zeit hindurch täglich studieren. Da dies durchzuführen beinahe unmöglich ist, müssen wir uns mit einer approximativen Schätzung der Absorption etc. des Quecksilbers durch Untersuchung des Urins begnïgen.

Nun, verhält es sich aber so, daß durch die Fäces eine bedeutende Quantität Hg ausgeschieden wird; das Verhältnis zwischen der durch Faeces und durch Urin eliminierten HgQuantität ist nicht konstant; sie wechselt nicht allein bei verschiedenen Personen, sondern auch bei ein und derselben Person an verschiedenen Tagen bei verschiedenen Gelegenheiten. Dies zeigt, daß man durch alleinige Untersuchung des Urins auf $\mathrm{Hg}$ zwar die durch den Urin eliminierte Hg-Menge in mehr oder weniger exakten ${ }^{2}$ ) Ziffern angeben kann, es gibt aber nicht die ganze aus dem Organismus eliminierte $\mathrm{Hg}$ Quantität in exakten Zahlen wieder, zeigt uns also nicht in exakten Zahlen, wie groß die Quecksilberabsorption gewesen ist.

Sicher ist, daß es für uns nicht von der geringsten Bedeutung ist, ob wir in exakten Zahlen angeben können, ob

1) Der Wert dieser sog. exakten Zahlen darf nicht überschätzt werden. Frl. Ratner hat in 18 Fällen eine genau abgewogene Quantität $\mathrm{Hg}$ einer Quantität Urin zugesetzt und gibt dic nach dem $\mathrm{F}$ arupschen Verfahren in diesem Urin nachgewiesene Quantität $\mathrm{Hg}$ an; in 7 von diesen 18 Fällen, d. h. in $39 \%$ der untersuchten Fälle hat sie eine größere Quantităt $\mathrm{Hg}$ gefunden und in sog. exakten Zahlen angegeben, als sie zugesetzt hat. 
sich im Urin z. B. $0.6 \mathrm{mg} \mathrm{Hg}$ befindet, oder ob derselbe 0.8 enthält; ron Bedeutung für uns ist es zu wissen, was diese Zahlen bedeuten, wenn wir die Absorption und Remanenz des Quecksilbers zu beurteilen versuchen wollen. In dieser Beziehung können wir uns nicht länger in exakten Zahlen ausdrücken, sondern müssen uns damit begnügen, uns auf Grund der sogenannten exakten Ziffern von Urinuntersuchungen ein approximatives Urteil darüber zu bilden. Finden wir z. B. am ersten Tage einer Injektion einen Hg-Gehalt ron 0.5 oder $1 \mathrm{mg}$, so können wir approximativ annehmen, daß nur eine geringe Quantität $\mathrm{Hg}$ absorbiert worden ist. Finden wir wiederum einen Hg-Gehalt von 6-8 mg im Urin, so können wir approximativ beurteilen, daß eine bedeutende $\mathrm{Hg}$-Quantität absorbiert worden ist - wie große diese Absorption aber wirklich gewesen ist, das können wir nicht einzig auf Grund der Urinuntersuchung wissen, oder in exakten Zablen ausdrücken, sondern wir müssen, wie ich es bei der Anwendung der Almén-Schillbergschen Methode gemacht habe, uns damit begnügen, uns einen approximativen Begriff darüber zu bilden, ob eine geringe, große oder bedeutende Quantität $\mathrm{Hg}$ absorbiert worden ist - und dies genügt vollständig für uns für den praktischen Zweck, um dessenwillen solche Untersuchungen (wenigstens von mir) ausgeführt worden sind.

Frl. Ratner stützt ihr Urteil auf die Untersuchung zweier Personen, die eine Injektion von $0^{\cdot 1} g$ Thymol-Hg bekommen haben; sie hat hierbei gefunden, daß die Hg-Elimination am ersten Tage 6.8 , resp. $8.2 \mathrm{mg}$, sowie am zweiten Tage $2 \cdot 5$, resp. $5 \cdot 7 \mathrm{mg}$ gewesen ist. Sie erwähnt auch zwei Fälle, die B ürgi untersucht hat und wo er eine Hg-Elimination am ersten Tage von 6.45 , resp. $6.4 \mathrm{mg}$, am zweiten Tage von 4.3 resp. $4 \cdot 4 \mathrm{mgm}$ und in einem dieser Fälle am dritten Tage eine von $2 \cdot 7 \mathrm{mg}$ gefunden hat.

Nach einer Kalomelinjektion von $0.05 g$ in zwei Fällen hat Frl. Ratner gefunden, daß die Hg-Elimination am ersten Tage 5.0 und $6.5 \mathrm{mg}$, am zweiten Tage 3.3 resp. $3.0 \mathrm{mg}$, sowie in dem einen Falle am dritten Tage $3.1 \mathrm{mg}$ gewesen ist. 
Im Verlaufe des Jahres 1908 habe ich einen Teil der Untersuchungen auf $\mathrm{Hg}$ im Urin nach der Injektion obiger sog. unlöslicher Hg-Präparate ausgeführt; ich habe diese jedoch nicht in der Absicht vorgenommen, den verschiedenen Typus für die Elimination des Quecksilbers nach diesen Injektionen zu studieren, sondern mein Zweck ist ein ganz anderer gewesen. Dies ist der Grund, weshalb ich in der Mehrzahl von Fällen keine Serienuntersuchungen Tag für Tag, nicht einmal für zwei aufeinander folgende Tage, d. h., die Anzahl Tage, während deren Frl. Ratner drei ihrer vier Fälle untersucht hat - nur einen Fall hat sie 3 Tage lang untersucht - aufweisen kann. Dadurch, daß ich an dem einen Tage eine Injektion mit dem einen Hg-Präparat und am folgenden Tage mit einem anderen Präparate gegeben habe, wie ich es in den meisten Fällen getan habe, ist der Kontrast zwischen der während dieser beiden Tage eleminierten $\mathrm{Hg}$-Quantität augenscheinlicher geworden und der Eliminationstypus für $\mathrm{Hg}$ ist bei diesen verschiedenen sog. unlöslichen $\mathrm{Hg}$-Präparaten deutlicher hervorgetreten.

Ich habe bei diesen Untersuchungen teils die AlménSchillbergsche, teils die Farupsche Methode angewendet; diese letzteren Untersuchungen sind von Herrn Apotheker Blom q rist ausgeführt worden.

Ich will nun zuerst die Fälle schildern, wo die AlménSchillbergsche Methode angewendet worden ist. Ich habe es für unnötig erachtet, die Anzahl und die Größe der in jedem Falle gefundenen $\mathrm{Hg}$-Kugeln zu beschreiben; ich führe deshalb beinahe in allen Fällen nur an, ob ich die $\mathrm{Hg}$-Quantität, in Übereinstimmung mit dem in meinem erwähnten Aufsatz rom Jahre 1886, als gering, groß, bedeutend usw. geschätzt habe.

In allen Fällen, wo die Injektion mit essigsaurem Thymolquecksilber, mit Kalomel und mit Sal.-Hg. gemacht wurde, sind 5 Zentigramm dieser Präparate (in Paraffinum liquidum 1:10 aufgeschlämmt) injiziert worden; von Ol. mercurioli ist 1 Teilstrich eingespritzt worden.

Fall I. 13./V. 6 Uhr Nachm. Injekt. von Thymol.-Hg. 14./V. $1 \mathrm{Uhr}$ Mittags im Urin sehr kleine Kugeln = gering. 11./V. 1 Uhr Mittags Inj. von Sal.-Hg. 14./V. 6 Uhr Nachm. eine große Menge größerer und kleinerer Kugeln $=$ bedeutend. 
Fall II. 27./V. Mittags Injekt. von Thymol.Hg. 28./V. 12 Uhr Mittags = geringe Hg-Menge im Urin. 28./5. $12 \mathrm{Uhr}$ Mittags Injekt. von Sal.-Hg. 28./V. 9 Uhr Nachm. = bedeutende Hg-Menge im Urin.

Fall III. 26./V. 1 Uhr 30 Min. Nachm. Inj. von Th.-Hg. 27./V. 12 Uhr Mittags $=$ keine Kugeln $=0$. 28./V. $12 \mathrm{Uhr}$ Mittags ein paar recht große Kugeln = gering. 29./V. $12 \mathrm{Uhr}$ Mittags mebrere, jedoch kleine Kugeln = gering.

Fall IV. 29./V. 2 Uhr Nachm. Inj. von Kalomel. 30./V. Abends = geringe Hg-Menge im Urin. 31./V. $2 \mathrm{Uhr}$ Nachm. lnj. von Sal.-Hg. 31./V. $9 \mathrm{Uhr}$ abends $=$ bedeutende $\mathrm{Hg}$-Menge im Urin.

F all V. 26./V. 1 Uhr 30 Min. Nachm. Inj. von Kalomel. 27./V. 7 Uhr Vorm. = geringe Hg-Menge im Urin. 28./V. $7 \mathrm{Uhr}$ Vorm. = geringe $\mathrm{Hg}$-Menge im Urin. 29./ $\mathrm{V} .7 \mathrm{Uhr}$ Vorm. = ziemlich große HgMenge im Urin. 29./V. $1 \mathrm{Uhr}$ Nachm. Inj, von Sal.HHg. 29./V. 6 Uhr Nachm. = bedeutende Hg-Menge im Urin.

Fall VI. 6./IV. Mittags Inj. von 0l. mercurioli. 7./IV. Morgens $=$ geringe $\mathrm{Hg}-\mathrm{Menge}$ im Urin. 7./IV. $12 \mathrm{Ubr} 30 \mathrm{Min}$. Nachm. Inj. von Sal.-Hg. 7./IV. 6 Uhr 30 Min. Nachm. = höchst bedeutende Hg-Menge im Urin.

Fall VII. 2./IV. Inj. von 0l. mercur. 3./IV. Morgens = sehr geringe Hg-Menge im Urin. 5./IV. 10 Uhr Vorm. Sal.-Hg. 5./IV. 2 Uhr $30 \mathrm{Min}$. Nachm. = höchst bedeutende Hg-Menge im Urin.

F all VIII. 11./IV. Inj. von 01. mereur. 12./IV. 5 Uhr Vorm. $=$ sehr geringe Hg-Menge im 'Urin. 12./IV. 2 Uhr 30 Min. Nachm. lnj, von Sal.-Hg. 12./IV. 7 Uhr. Nachm. = höchst bedeutende Hg-Menge im Urin.

F all IX. 15./IV. 6 Uhr Nachm. Inj. von Ol mercur. 16./IV. $12 \mathrm{Uhr}$ 20 Min. Vorm. = sehr geringe Hg-Menge im Urin. 16./IV. 1 Uhr Nachm. Inj. von Sal.-Hg. 16./IV. $7 \mathrm{Uhr}$ Nachm, = höchst bedeutende Hg-Menge im Urin.

Fall X. 17./IV. 2 Uhr 30 Min. Nachm. Inj. von Ol. merc. 18./IV. $12 \mathrm{Uhr} 45 \mathrm{Min} . \mathrm{Nachm}$. = sehr geringe Hg-Menge im Urin. 18./IV. $1 \mathrm{Uhr}$ 30 Min. Nachm. Inj. von Sal.-Hg. 18./IV. 8 Jhr Nachm. = höchst bedeutende $\mathrm{Hg}$-Menge im Urin.

F all XI. 18./IV. Inj. von Ol. merc. 23./IV. 5 Uhr 30 Min. Vorm. $=$ geringe $\mathrm{Hg}$-Menge im Urin. 23./IV. $6 \mathrm{Uhr}$ Nachm. Inj. von Sal.-Hg. 24./4. Morgens $=$ bedeutende Hg-Menge im Urin.

Es ist also in sämtlichen Fällen am ersten Tage eine geringe Quantität $\mathrm{Hg}$ eliminiert worden, ob nun Thymol-Hg, Kalomel oder 01 . mercurioli eingespritzt worden ist. In einem Falle, wo Thymol-Hg und in einem Falle, wo Kalomel injiziert worden ist, wurden auch am zweiten und dritten Tage nach 
Noch einige Worte üb. d. Elimination des Quecksilbers etc. 171

der Injektion Hg-Untersuchungen vorgenommen; auch während dieser Tage erwies sich die eliminierte Quantität Quecksilber als unbedeutend.

Wenn wiederum Salizyl-Hg eingespritzt wurde, d. h. in 10 Fällen, war in jedem dieser Fälle die eleminierte Hg-Quantität bedeutend oder höchst bedeutend und zwar schon einige Stunden nach erfolgter Injektion.

Sämtliche diese Untersuchungen weisen mit Bestimmtheit darauf hin, daß der Typus für die Hg-Elimination bei der Injektion dieser schwerlöslichen Präparate ein ganz verschiedener ist und $\mathrm{daB}$ der erst von Lindén für Sal.-Hg und dann von B ürgi für sämtliche schwerlösliche $\mathrm{Hg}$-Präparate aufgestellte Eliminationstypus nur für Salizyl-Hg gilt.

Ich versuchte nun noch einmal zu prüfen, ob ich mit dem Farupschen Verfahren zu einem anderen Resultat kommen würde, als dem, zu dem ich zuerst mit demselben gekommen war. Ich untersuchte zu diesem Zwecke 6 Personen. In diesen Fällen habe ich zuerst Thymolquecksilber oder Kalomel injiziert und nach Verlauf von drei Tagen eine Injektion mit Sal.Hg gemacht. Ein solcher Versuch mit 0l. mercurioli erschien mir vollständig überflüssig, da von B lo mq v i st eine sehr große Anzahl Untersuchungen mit diesem Mittel gemacht worden waren, die einstimmig gezeigt hatten, daß nach der Injektion dieses in den ersten Tagen konstant eine sehr kleine Quantität $\mathrm{Hg}$ eliminiert wurde.

In zwèi Fällen habe ich 5 Zentigramm injiziert, in dem einen Thymol-Hg, in dem anderen Kalomel, sowie nach 3 Tagen 5 Zentigramm Sal. Hg eingespritzt.

Fall XII. 17./IX. Inj. von Th.-Hg. 17,-18./IX. wurden durch den Urin $840 \mathrm{~g} 0.4 \mathrm{mg} \mathrm{Hg}$ eliminiert. 18.-19./LX. wurden durch den Urin $1115 \mathrm{~g} 1.2 \mathrm{mg} \mathrm{Hg}$ eliminiert. 19.-20./IX. wurden durch den Urin $1030 \mathrm{~g} \mathrm{2 \cdot 4} \mathrm{mg} \mathrm{Hg}$ eliminiert. 20./IX. Inj. von Sal.-Hg. 20.-21./IX. wurden durch den Urin $1055 \mathrm{~g} 5^{*} 4 \mathrm{mg}$ Hg eliminiert.

Fal1 XIII. 17./IX. Inj, von Kalomel. 17.-18./IX. wurden durch $1285 \mathrm{~g}$ Urin kein $\mathrm{Hg}$ eliminiert. 18. - 19./IX. wurden durch den Urin $1030 \mathrm{~g} 1.0 \mathrm{mg} \mathrm{Hg}$ eliminiert. 19.-20./1X. warden durch den Urin $1480 \mathrm{~g}$ 
1.8 mg Hg eliminiert. 20./IX. Inj. von Sal.-Hg. 20.-.21./IX. wurden durch den Urin $1260 \mathrm{~g} 3.6 \mathrm{mg} \mathrm{Hg}$ eliminiert.

In den folgenden Fällen wurden $10 \mathrm{cg}$ Tbymol-Hg, resp. Kalomel und drei Tage danach 5 og Sal.-Hg injiziert.

Fall XIV. 2./IX, Inj, von Thym./Hg. 2.-3./IX. wurden durch den Urin, $860 \mathrm{~g}, 0.8 \mathrm{mg} \mathrm{Hg}$ eliminiert. 3.-4./1X. wurden durch den Urin, $1150 \mathrm{~g}, 0.5 \mathrm{mg} \mathrm{Hg}$ eliminiert. 4.-5./IX. wurden durch den Urin, $1320 \mathrm{~g}$, $0.9 \mathrm{mg} \mathrm{Hg}$ eliminiert. 5./IX. Inj. von Sal.-Hg. 5.-6./IX. wurden durch den Urin, $1115 \mathrm{~g}, 1.8 \mathrm{mg} \mathrm{Hg}$ eliminiert.

F'all XV. 8./IX. Inj, von Kalomel. 8.-9./IX. wurden durch den Urin, $1340 \mathrm{~g}, 0.5 \mathrm{mg} \mathrm{Hg}$ eliminiert. 9.-10./IX. wurden durch den Urin, $1165 \mathrm{~g}, 0.7 \mathrm{mg}$ Hg eliminiert. 10.-11./IX. wurden dureh den Urin, $1450 \mathrm{~g}$, $1.3 \mathrm{mg}$ eliminiert. 11./IX. Inj. von Sal.-Hg. 11.-12./IX. wurden durch den Urin, $1660 \mathrm{~g}, 62 \mathrm{mg} \mathrm{Hg}$ eliminiert.

Schließlich wurden in zwei Fällen je $5 \mathrm{cg}$ Thymol-Hg und Kalomel sowie drei Tage darauf $10 \mathrm{eg}$ Sal.-Hg injiziert.

F all XVI. 26./VIII. Inj. von Thymol-Hg. 26.-27.0VIII. wurden durch den Urin, $890 \mathrm{~g}, 1 \cdot 1 \mathrm{mg} \mathrm{Hg}$ eliminiert. 27-28./VIII. wurden durch den Urin, $840 \mathrm{~g}, 1.1 \mathrm{mg}$ eliminiert. 28.-29./VIII. wurden durch den Urin, $1050 \mathrm{~g}, 1.2 \mathrm{mg} \mathrm{Hg}$ eliminiert. 29./VIII. Inj. von Sal.-Hg. 29.-30./VIII. wurden durch den Urin, $1410 \mathrm{~g}, 8.0 \mathrm{mg} \mathrm{Hg}$ eliminiert.

Fall XVII. 20./VIII. Inj. von Kalomel. 20.-21./VIII. wurden durch den Urin, $770 \mathrm{~g}, 0.4 \mathrm{mg} \mathrm{Hg}$ eliminiert. 21.-22./VIII. wurden durch den Urin, $575 \mathrm{~g}, 0.8 \mathrm{mg} \mathrm{Hg}$ eliminiert. 22.-23./VIII. wurden durch den Urin, $795 \mathrm{~g}, 1.4 \mathrm{mg} \mathrm{Hg}$ eliminiert. 23./VIII. Inj. von Sal.-Hg. 23.-24./VIII. wurden durch den Urin, $670 \mathrm{~g}, 8.1 \mathrm{mg} \mathrm{Hg}$ eleminiert.

Wir finden somit in allen diesen Fällen, ob nun Thymol$\mathrm{Hg}$ oder Kalomel eingespritzt wurde, ob die eingespritzte Dosis 5 oder 10 Zentigramm gewesen ist, am ersten Tage eine geringe $\mathrm{Hg}$-Elimination, die in den beiden folgenden Tagen allmählich steigt, während die eliminierte Hg-Quantität am vierten Tage, wo die Injektion mit Sal.-Hg gemacht wurde, in allen Fällen steigt, und in allen, außer einem, sogar bedeutend steigt. Also konnte auch mit dem Farupschen Verfahren der von Bürgi und Frl. Ratner observierte Typus für die Hg-Elimination nicht beobachtet werden.

Die Ursache der verschiedenen Resultate zwischen diesen und den in $B$ ür $\mathrm{g}$ is Laboratorium ausgeführten Untersuchungen 
Noch einige Worte üb. d. Elimination des Quecksilbers etc. 173

kann ich nicht erklären. Dagegen habe ich aber die hier sowohl nach der Almén-Schillbergschen Methode wie nach dem Far u pschen Verfahren gemachten Untersuchungen vollständig mit einander übereinstimmend gefunden, und da sie mit den von mir 1906 veröffentlichten Untersuchungen und auch mit zwei anderen, hier nicht aufgenommenen, kürzlich untersuchten Fällen übereinstimmen, kann ich natürlich meine Auffassung, daß der Typus für die Elimination von $\mathrm{Hg}$ nach der Injektion von Sal..Hg dem Typus für die Elimination des Quecksilbers nach der Injektion von Thymol-Hg und Kalomel sowie nach der von Ol. mercurioli ganz ungleich ist, nicht deswegen ändern, weil Bürgi und Frl. Ratner zu einem anderen Resultate, als ich, gekommen sind.

Ich kann hier einen, wenn auch indirekten Beweis für den ungleichen Eliminationstypus liefern, nämlich die ganz verschiedene Reaktion, die ganz verschiedene Steigerung der Hyperämie bei syphilitischen Roseolaflecken, die nach der ersten Injektion von Sal.-Hg und nach der ersten Injektion von Thymol$\mathrm{Hg}$, Kalomel oder 0l. mercurioli auftritt. Ich will hier nur auf dieses Verhältnis, das in vollkommener Übereinstimmung mit dem verschiedenen Absorptions-(resp. Eliminations-)Typus des $\mathrm{Hg}$ nach der Injektion mit diesen verschiedenen $\mathrm{Hg}$-Präparaten steht, aufmerksam machen. (Siehe meinen Aufsatz hierüber in diesem Archiv, Bd. XCV, Heft 1.)

Schließlich will ich noch einen Beweis vorbringen.

Frl. Ratner schreibt, „dal es gar nicht einzusehen ist, warum ein Präparat, wie das Hydr. Thymolo-Aceticum sich im Organismus so wesentlich anders verhalten sollte, als das Hydr. Salicylicum". Meine Erklärung hiefür, nämlich daß SaL.-Hg in eiweißhaltigen Flüssigkeiten bedeutend löslicher ist, als ThymolHg und Kalomel, hat sie nicht akzeptabel gefunden. Ich habe zwar gezeigt, daß dies sich in der Ascitesflüssigkeit, in der Ovarialflüssigkeit so verhält und $\mathrm{da} ß$ es im Blutserum noch mehr der Fall ist; den Wert dieser Untersuchungen weist aber Frl. Ratner damit ab, daß, sie über das Verhalten der ge- 
nannten Präparate im lebenden Organismus recht wenig aussagen". Dieses ihr Raisonnement erscheint mir eigentïmlich, denn sonst hat sich immer die Auffassung geltend gemacht, daß man für die Injektion von $\mathrm{Hg}$-Präparaten solche zu finden suchen muß, die sich in eiweißhaltigen Flüssigkeiten, somit auch in den Körpersäften, so leicht wie möglich löslich halten können, weil sie hiedurch leichter absorbiert werden können. Deshalb hat man ja darnach gestrebt, Quecksilberalbuminatverbindungen für die Injektion herzustellen, deshalb setzt man ja auch den Sublimatlösungen Kochsalz zu, wenn man sie zur Injektion anwenden will. Es ist wohl niemand auf die Idee gekommen, das in eiweißhaltigen Flüssigkeiten unlösliche Zinnober zur Injektion anzuwenden, während es dagegen, wie andere in eiweißhaltigen Flüssigkeiten unlösliche Präparate, sich ganz vortrefflich für die Tätowierung eignet, wo die Absicht ist, daß es für die Zukunft unzersetzt verbleiben soll.

Es scheint mir indessen klar zu sein, daß ein im Blutserum, in den Körpersäften leicht löslicher Stoff leichter absorbiert werde, als ein Stoff, der in diesen eiweißhaltigen Flüssigkeiten schwerlöslich ist, was natürlich nicht hindert, daß auch der letztere Stoff auf diese oder jene Weise, obschon allmählich, so zersetzt werden kann, daß er absorbiert wird. Wenn diese Anschauung richtig ist, dürfte man dies bei der Untersuchung der resp. Depots nach der Injektion verschiederer HgPräparate finden können. Eine solche Untersuchung an Menschen zu machen, dürften wir äußerst selten Gelegenheit haben; in ein paar Fällen (bei Obduktionen) habe ich gefunden, wie das in Eiweißflüssigkeiten sehr schwer lösliche Merkuriolöl mehrere Wochen nach erfolgter Injektion noch zum großen Teil an dem Platz, wo es eingespritzt worden ist, wenn auch, wenigstens teilweise, verändert, gelegen hat. Tierversuche könnten uns hierüber ganz gute Aufschlïsse geben, und ich beabsichtige auch solche zu machen, wenn sie auch nicht in allen Beziehungen auf Menschen anwendbar sind.

Um wenigstens eine Vorstellung davon zu bekommen, wie lange die Depots nach der Injektion dieser verschiedenen sog. schwerlöslichen $\mathrm{Hg}$-Präparate im Körper bleiben, wollte ich versuchen durch Röntgenphotographien Aufschlüsse hierüber 
Noch einige Worte üb. d. Elimination des Quecksilbers etc. 175

zu erhalten. A priori besteht ja, wenn die verschiedene Löslichkeit der verschiedenen $\mathrm{Hg}$-Präparate in eiweißhaltigen Flüssigkeiten auch im Organismus Geltung haben sollte, die größte Wahrscheinlichkeit dafür, daß ein Injektionsherd nach Salizyl-Quecksilber schneller verschwindet, als ein Herd nach Thymol-Hg oder Kalomel oder Ol. mercurioli. Um dies zu erforschen, habe ich mit diesen Hg-Präparaten einen Teil Injektionen gemacht, und der Vorsteher des Röntgeninstitutes des Königl. Serafimerlazarettes, Dr. Gösta F or s s e ll, hat die Freundlichkeit gehabt, diese Injektionsherde zu photographieren, wofür ich ihm hier meinen Dank abstatte.

Es erwies sich sofort beinahe unmöglich, von den intramuskulären Injektionsherden an den Stellen, wo die Injektionen jetzt gewöhnlich angebracht werden, deutliche Röntgenphotographien zu erhalten; in allen diesen Versuchen sind die Injektionen deshalb sublutan gemacht worden.

Von jedem dieser vier sog. unlöslichen Hg-Präparate wurde in ein zusammengepreßtes Baumwollebäuschchen eine Einspritzung von derselben Menge und derselben Beschaffenheit, wie sie dann in das Gesäß gemacht wurde, vorgenommen; nach allen diesen in die Baumwolle eingespritzten Hg-Präparaten wurden bei der Röntgenphotographierung scharf und deutlich begrenzte Herde sichtbar. Einer Person wurde in der Klinik $0.5 \mathrm{~g}$ Paraffinum liquidum ohne Zusatz irgendwelches Hg-Präparates eingespritzt; bei der Photographierung am folgenden Tage war nicht die geringste Spur einer Injektion nachweisbar; die nach der Injektion von in Paraffinum liquidum aufgeschlämmten $\mathrm{Hg}$-Präparaten entdeckten Herde müssen folglich ausschließlich auf jenen beruhen. Ich will auch erwähnen, daß in einem Falle $1 g$ 1\% Sublimatlösung mit dem gewöhnlichen Zusatz von Kochsalz eingespritzt worden ist; nach dieser Einspritznng konnte am folgenden Tage bei der Röntgenphotographierung kein Herd wahrgenommen werden.

Von Ol. mercurioli ist ein Teilstrich ( $\mathrm{L}$ a $\mathrm{ng}$-Spritze) eingespritzt worden; von Kalomel, Thymol-Hg und Salizyl-Hg sind $5 \mathrm{cg}$ in $0.5 \mathrm{~g}$ Paraffinum liquidum aufgeschlämmt, außer in einem Falle, wo nur $3 \mathrm{cg}$ eingespritzt worden sind, injiziert worden. Sämtliche Injektionen sind, außer einer, die am Ober- 
arm vorgenommen wurde, im Gesäß gemacht. In dem Gedanken, $\mathrm{da} B$ die eingespritzte $\mathrm{Hg}$-Masse dort leichter abgebildet werden könnte, ließ ich in meinen linken Oberarm eine subkutane Injektion von $5 \mathrm{cg}$ Sal.-Hg machen; das Resultat war genau dasselbe, wie bei der Injektion in die Nates; es kann von Interesse sein, zu erwähnen, daß die Absorption des Sal.-Hg bei mir, trotz meiner bald 63 Jahre, ebenso rasch und vollständig vor sich ging, wie die Absorption bei den anderen Sal.-Hg-Injektionen, trotzdem diese an jugendlichen, gesunden Individuen vorgenommen worden sind.

G. Forssell hat folgenden Bericht über die Röntgenuntersuchungen erstattet.

Im Auftrage des Herrn Professor E. Welander habe ich die Resorptionsgeschwindigkeit gewisser Quecksilbersalze, d. h. ihren Übergang im Organismus von unlöslichen Verbindungen zu löslichen röntgenographisch kontrolliert.

Die Technik bei den Röntgenuntersuchungen ist folgende gewesen: Die von Herrn Prof. Welander ausgeführten Injektionen sind nach meiner Anweisung innerhalb des höchsten Teiles der Nates auf solche Weise placiert worden, daß das Bild des injizierten Quecksilbersalzes von der Seite her auf einer in der Rima ani angebrachten Photographieplatte projiziert werden konnte. Der Pat. hat bei der Röntgenphotographierung mit gut hochgezogenen Knien auf der Seite gelegen. Die Photographieplatte ist, auf Holzklötze gestïtzt, tief in die Rima ani hineingedrückt und mittels eines auf dem hervorragenden Teile der Platte angebrachten Gewichtes fixiert worden. Eine Kompressionsblende von $10 \mathrm{~cm}$ Durchmesser ist unter leichtem Druck über die Partie, wo die Injektion ausgeführt wurde, eingestellt worden. Für alle Röntgenogramme ist dieselbe Röhre (Müller 13a) angewendet worden, welche zu demselben Härtegrad (W alter 4-5) reguliert und mit derselben sekundären Stromstärke (31\% Mil. Amp.) getrieben worden ist. Die Expositionszeit ist für alle Fälle 12 Sekunden und der Antikathodenabstand $50-52 \mathrm{~cm}$ gewesen.

Von den untersuchten Quecksilbersalzen Merkuriol, Kalomel, Acetothymolas hydrargyricus und Salicylas hydrargyricus ist ein Röntgenogramm ausgefiihrt worden, wo diese Salze in 
Noch einige Worte üb. d. Elimination des Quecksilbers etc.

den angewendeten Dosen, in fette Watte injiziert, nebeneinander dargestellt sind. Dieses Bild zeigt, dab das Merkuriol einen dichteren Schatten als die übrige gibt, welche Injektionspräparate das Röntgenlicht ungefähr gleich stark absorbiert haben. Serien von Injektionen mit den erwähnten Salzen sind röntgenographiert worden.

\section{0l. Merkurioli.}

Elsa E., $17 \mathrm{Jahr}, 0.067 \mathrm{~g}$ Merkuriol (= I Teilstrich Ol. Mercurioli), rechte Seite, Inj. den 23. Sept., 1 Uhr nachm.

Röntgenographierungen den 24., 26. und 28. Sept, sowie den 3. u. 16. Okt. Am 24. Sept. waren ein dichter Schatten von runder Form und von ungefähr $5 \mathrm{~mm}$ Durchmesser mit streifenförmigen Ausläufern nach oben und unten, sowie einige kleinere Schatten im Stichkanal sichtbar. Nach drei Tagen (26./IX.) hatte sich der Schatten deutlich vermindert, war aber fortdauernd von großer Dichtigkeit; ungefähr dasselbe Aussehen nach 5 Tagen (28./IX.). Nach 10 Tagen (3./X.) nabm der Schatten etwa ein Drittel seines ursprünglichen Umfanges ein, der Rest ist aber noch immer zusammenhängend und dicht; er zeigt zahlreiche, dünne, streifenförmige Ausläufer. Noch nach $23 \mathrm{Tagen}(16 . / \mathrm{X}$.) ist ein deatlicher, aus kleinen, dichten, strichförmigen Schatten zusammengesetzter Schatten auf einem $8 \times 5 \mathrm{~mm}$ großen Gebiet vorhanden.

\section{Kalomel.}

Elsa E., 17 Jahre. Linke Seite. 0.05 g Kalomel, Inj. den 23. September 1908.

Die Röntgenographierungen sind zu denselben Zeiten wie beim Merkuriolversuche ausgeführt.

Das Kalomel gab einen tropfenförmigen, dichten Schatten von $6 \times 9 \mathrm{~mm}$ Ausdehnung, welcher noch nach 5 Tagen nur unbedeutend verschwunden war. Nach 10 Tagen hatte sich der Schatten ungefähr um die Hälfte seiner Größe vermindert, der Rest war aber von bedeutender Dichtigkeit. Nach 23 Tagen war nur ein $4 \times 1 \mathrm{~mm}$ grober Schatten vorhanden, welcher jedoch noch vollständig deutlich war.

\section{Aceto-thymol. Hydrarg.}

Birger H., 20 Jahre. Linke Seite. 0.05 $g$ Aceto-thymol. Hydrarg. Inj. 2. Okt.

Das Röntgenogramm zeigte am 3. Okt. einen flockigen, stellenweise dichteren Schatten mit dünnen, über ein Gebiet von etwa $7 \times 15 \mathrm{~mm}$ ausgebreiteten Råndern. Spätere Röntgenogramme am 16., 17., 19., 23. und 26. Oktober, das letzte also nach $23 \mathrm{Tagen}$, zeigen nur eine geringe Verdünnung des Schattens. 


\section{Salicylas hydrargyricus.}

Birger, H. - Rechte Seite. Inj. $0.05 \mathrm{~g}$ Salicyl. hydrargyr. am 16.10, 12 Uhr 30 Min. Nachm.

Bei der Röntgenographierung $1 \frac{1}{2}$ Stunden nach -der Injektion zeigte sich ein dichter, über ein etwa $12 \times 8 \mathrm{~mm}$ großes Gebiet verteilter Schatten, der eine kleinere äußere und eine mit dieser durch eine schmale Brücke zusammenhängende innere Partie aufweist. Am folgenden Tag war der Schatten kleiner und hatte deutlich an Dichtigkeit abgenommen. Am dritten Tage (19./X.) war dieser äußere Schatten beinahe verschwunden und der innere hatte sich noch mehr verdünnt. Nach 7 Tagen war der änßereSchattenvollständigverschwunden und der innere mit Schwierigkeit bemerkbar. Nach 10 Tagen (26./X.) waren beide Schatten verschwunden.

E. W. - Injektion von $0.05 \mathrm{~g}$ Salic. hydrarg. in den linken Oberarm, am 11./X.

Das Röntgenogramm am 12./X. zeigte einen deutlichen, obschon dünnen Schatten. Am 14./X. war der Schatten an den meisten Stellen dünner, ohne deutliche Grenze. 19./X. Der Schatten ist nach 8 Tagen vollständig verschwunden.

Gleichzeitige Injektion von Salic. hydr. und aceto-thymol hydrarg. nebeneinander.

Viktor L., 25 Jahre, $0.03 g$ Salicyl. hydrarg. und ebenso viel acetothym. hydrarg. wurden in etwa $4 \mathrm{~cm}$ Abstand voneinander injiziert und die Injektionsstellen bezeichnet (8./X.) Am folgenden Tage (9./X.) gab das aceto-thymol. hydrargyr. einen dichten, bohnengroßen Schatten; am Platze der Injektion von Salicyl. hydrargyr. war nur ein unbedeutender streifenförmiger Schatten zu entlecken.

Kurz nach der Injektion einer löslichen Quecksilbersalzes, Sublimates, wurde ein Radiogramm aufgenommen; hierbei wurde kein begrenzter Schatten erhalten, das Gewebe auf dem Gebiete der Infiltration und der umgebenden Anschwellung erschien diffus dichter, wie bei einer gewöhnlichen ödematösen Anschwellung. Eine Injektion von nur reinem Paraffin zeichnete sich nicht ab.

Stockholm, den 18. Nov. 1908.

Gösta Forssell.

Die Röntgenbilder sprechen so für sich selbst, daß ein Kommentar nicht pötig ist. Die Bilder des Sal.-Hg unterscheiden sich vollständig von den Bildern der übrigen $\mathrm{Hg}$-Präparate. In den Bildern nach diesen sehen wir noch 23 Tage nach erfolgter Injektion deutliche Herde zurückbleiben; nach der Injektion von Sal.-Hg sind die Herde nach 8-10 Tagen, sogar in meinem Oberarm, vollständig verschwanden und es läßt 
Noch einige Worte üb. d. Elimination des Quecksilbers etc. 179

sich nicht der kleinste Schatten nach der Injektion entdecken. Was bedeutet nun dies? Natürlich bedeutet es, daB die Absorption des injizierten Sal.-Hg ganz anders vor sich geht, als die Absorption der anderen sog. unlöslichen $\mathrm{Hg}$-Präparate, und zwar in voller Übereinstimmung mit dem, was wir durch die Untersuchungen auf $\mathrm{Hg}$ im Urin nach der Einspritzung dieser verschiedenen Hg-Präparate gelernt haben.

Summa summarum finden wir also, daß $\mathrm{Hg}$-Untersuchungen des Urins nach der Almén-Schillbergschen Methode, daß solche nach dem Farupschen Verfahren, daß die Hyperämie an und um die makulösen Syphilide, die am ersten Tage nach der Injektion von Sal.-Hg, aber nicht nach der Injektion der anderen schwer löslichen $\mathrm{Hg}$-Präparate auftritt, wie daß die Röntgenabbildungen nach diesen Injektionen, die zeigen, daß die Herde sich nach der Injektion von Sal.-Hg bedeutend rascher verkleinern und verschwinden, als die Herde nach der Injektion von Kalomel, Thymol-Hg und Ol. mercurioli - wir finden, $\mathrm{daB}$ alles dieses ein und dieselbe Sprache spricht, nämlich $\mathrm{da} B$ die Hg-Absorption und die davon abhängige $\mathrm{Hg}$-Elimination nach der Injektion von Sal.-Hg auf eine ganz andere Weise, nach einem ganz anderen Typus vor sich geht, als nach der Injektion von Kalomel, Thymol-Hg und Ol. mercurioli. Ich kann es nicht anders verstehen, als daB dies damit im Zusammenhang stehen muß, daß das Salizylquecksilber in eiweißhaltigen Flüssigkeiten, in Körpersäften viel leichter löslich ist, als die drei anderen sog. unlöslichen Hg-Präparate.

Ich (persönlich) muß es für erwiesen halten, daß der Eliminationstypus von $\mathrm{Hg}$ nach der Injektion von Sal.-Hg ein ganz anderer ist, als der Eliminationstypus nach der Injektion. der übrigen sog. unlöslichen Hg-Präparate.

In therapeutischer Beziehung ist dies von großer praktischer Bedeutung und dies ist der Grund, daß ich noch einmal auf verschiedene Weise Beweise hierfür zu geben versucht habe. 
Die Erklärung der Abbildungen auf Taf. $V-$ - $X$ ist de $m$ Texte zu entnehmen. 


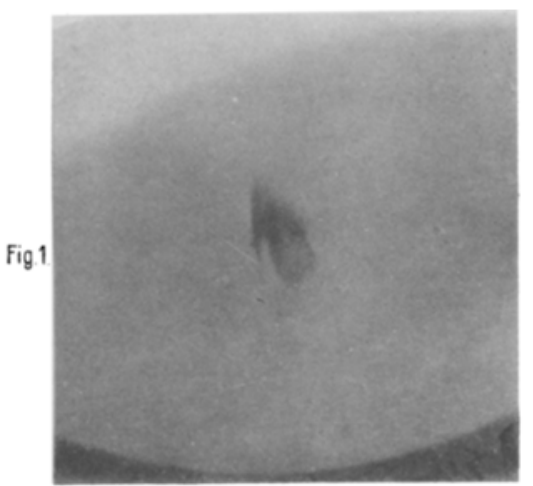

24. $1 \mathrm{x}$.

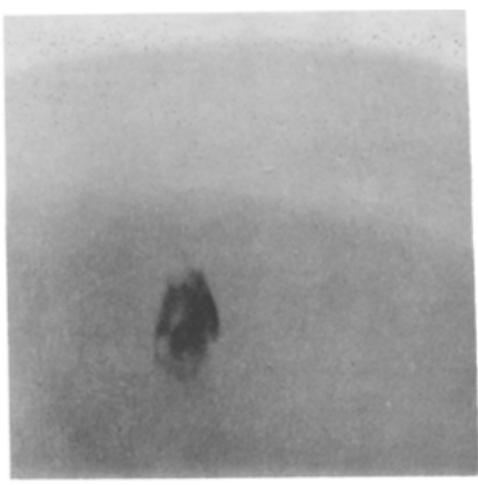

26. / IX

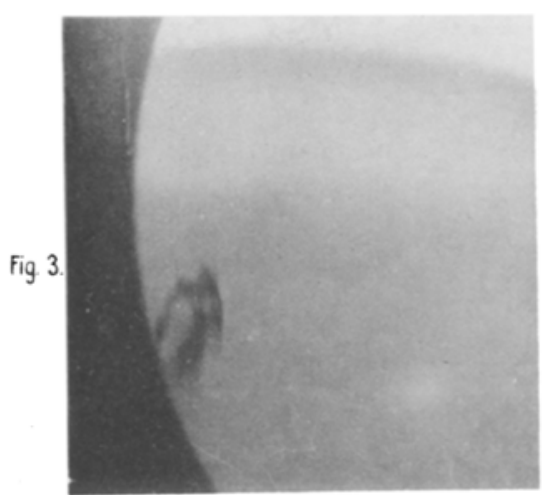

28/ IX.

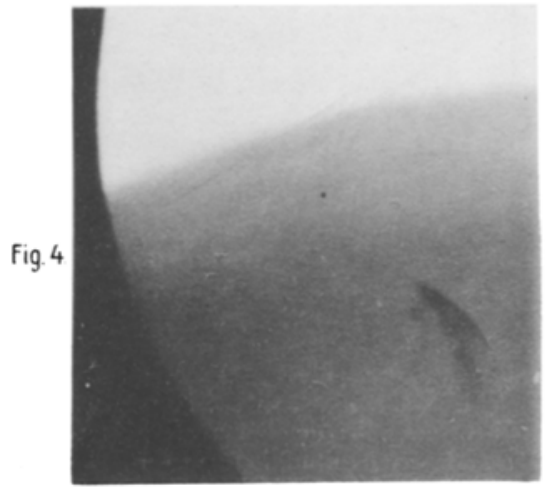

3. $\mathrm{X}$

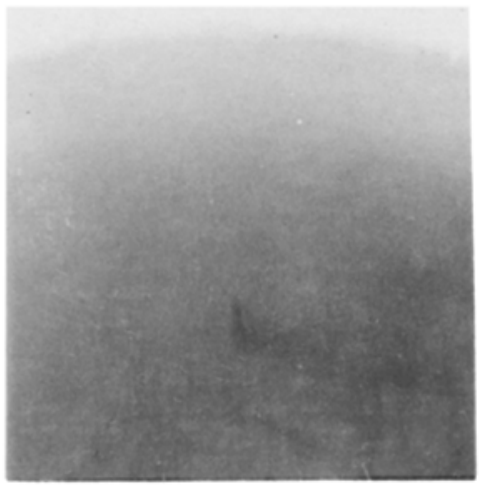

$16 / X$

E.Engberg Kalomel.

Welander : Elimination des Quecksilbers. 
Archiv f. Dermatologie u.Syphilis Band XCVI.

TAF. VI.

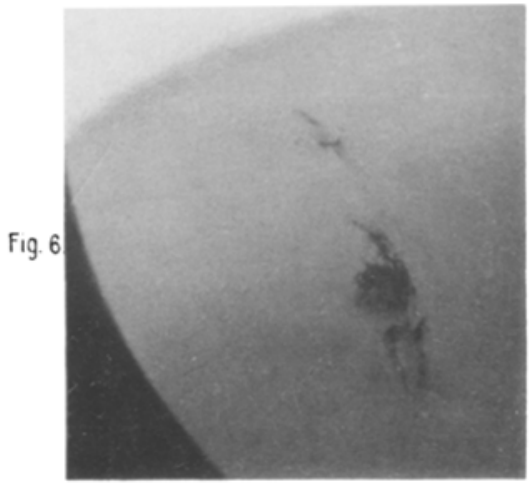

24. IX.

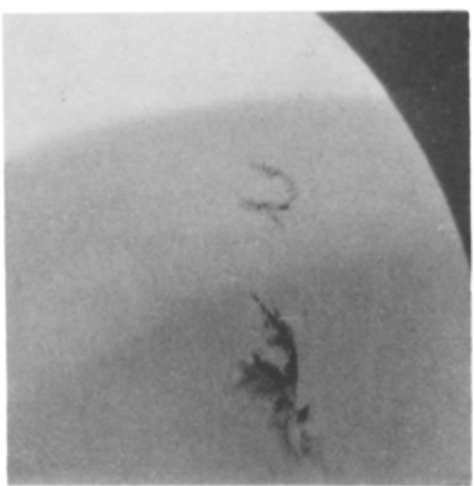

26. IX

fig 7.

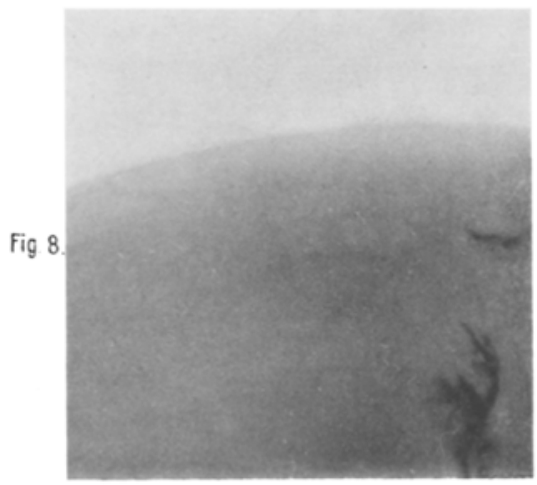

28. IX.
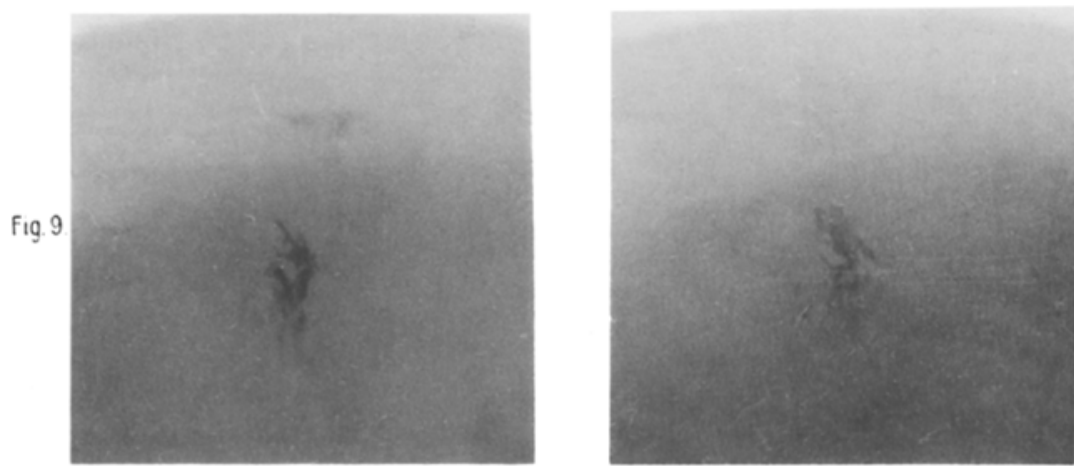

3. $\mathrm{x}$

$16 / X$

Welander : Elimination des Quecksilbers. 


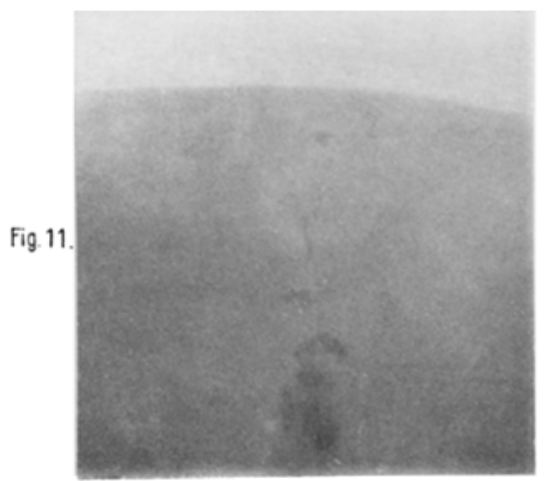

3. $x$

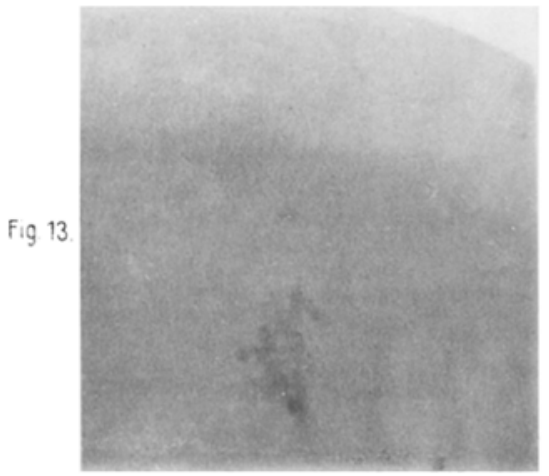

17. $X$

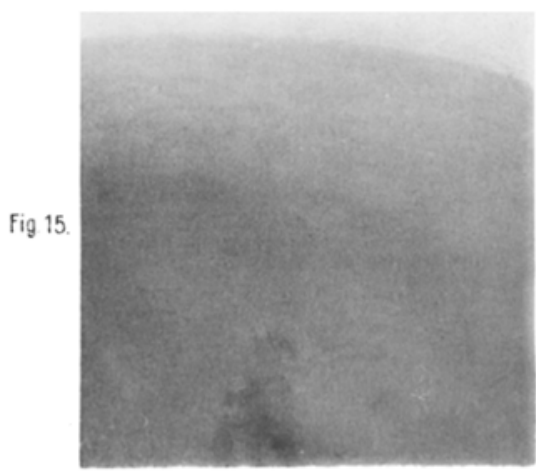

$33 \times$ Birger Hultmann. Aceto-thymol hydrarg

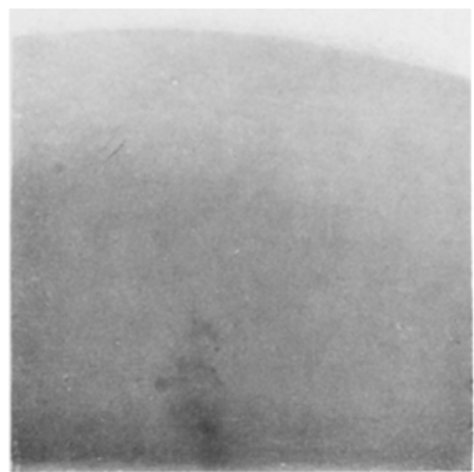

$16 / x$

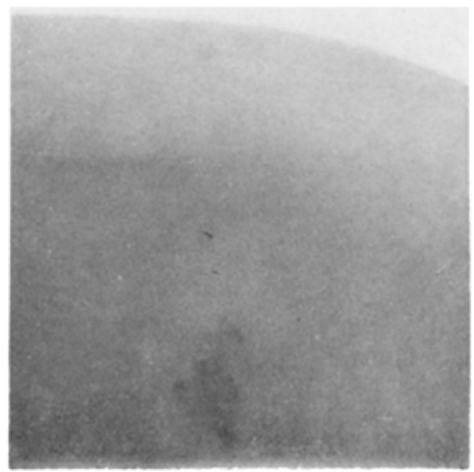

19. $\mathrm{X}$

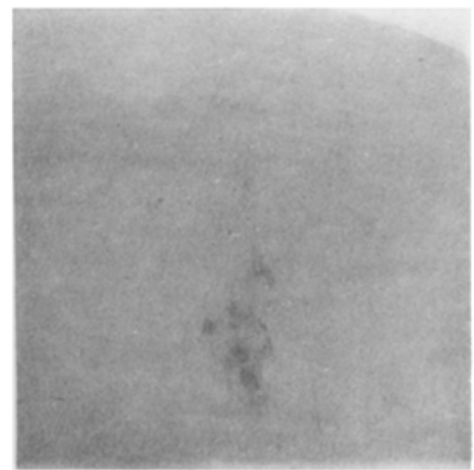

26. $x$
Fig 12

Fig 14.

fig. 16

Welander : Klinination des Quecksilbers. 


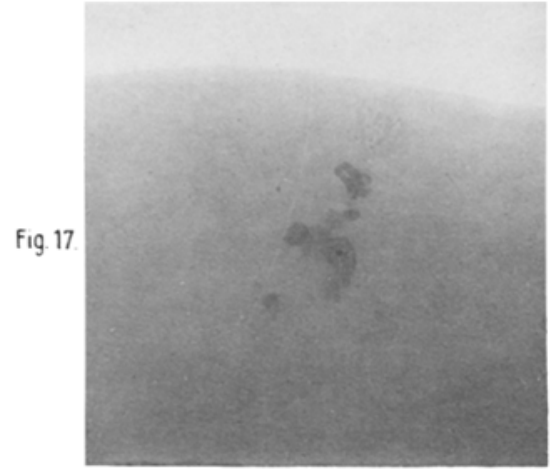

$16 / x$

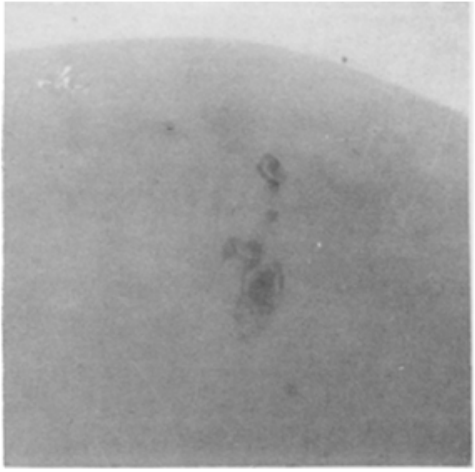

17. $\mathrm{X}$

Fig 18

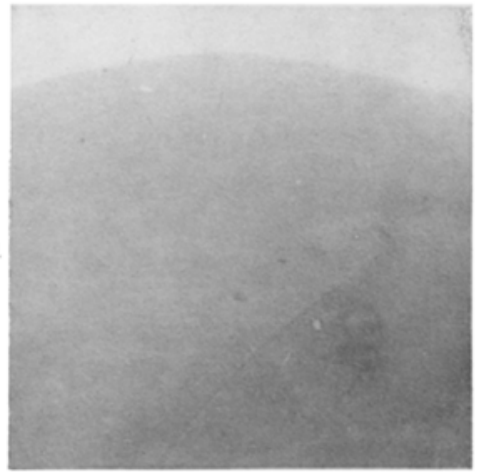

$19 / x$

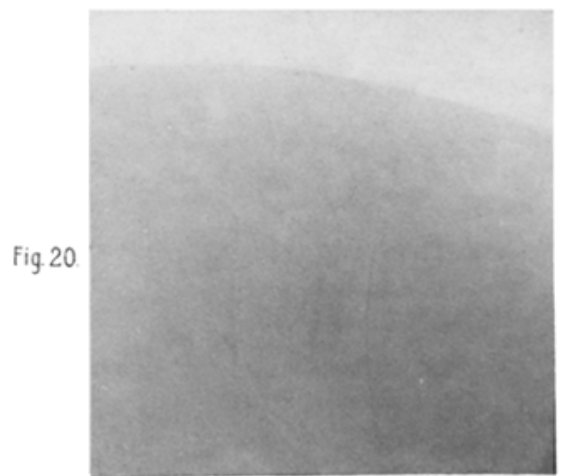

23. $\mathrm{x}$

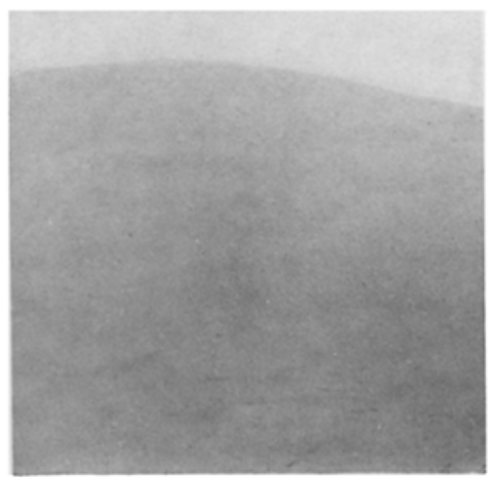

26. $/ x$

Birger Hultmann. Salıcyl hydrarg

Welander : Elimination des Quecksilbers.

fig. 21 .

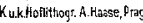


Fig. 22 .

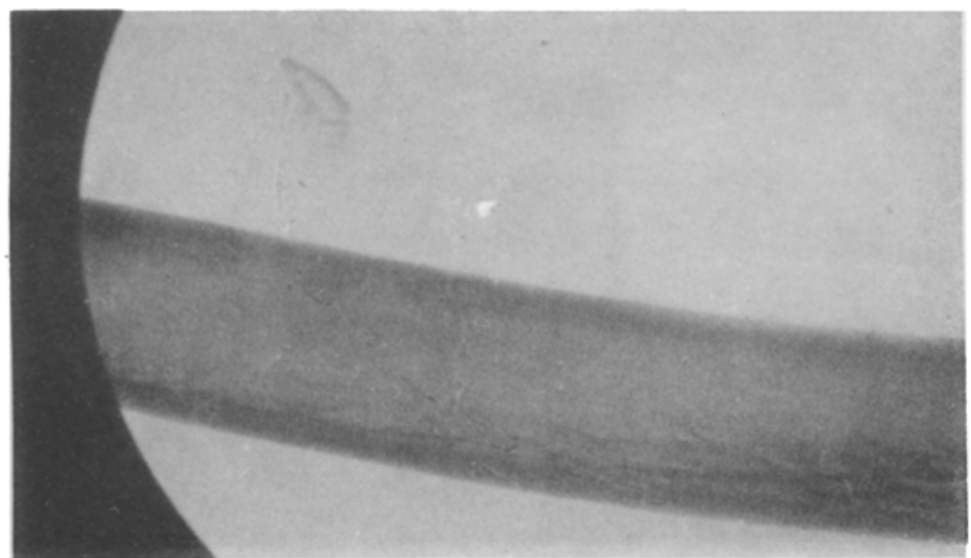

14. $x$

Fig. 23

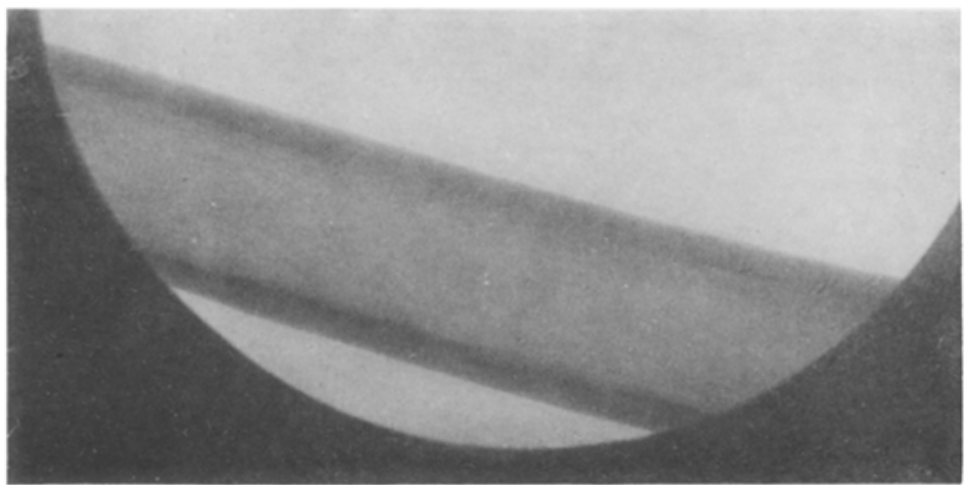

19. X E.Welander. Salicyl hydrang.

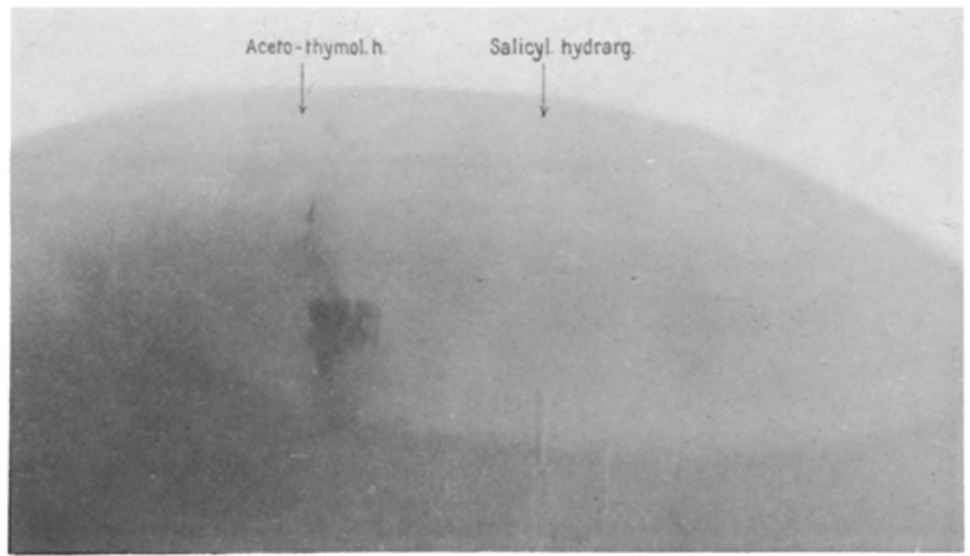

fig. 24

9. X. Viktor Larson. Acerothymol und salicyl hydrarg neben einander

Welander : Flimination des Quecksilbers. 